Check for updates

Cite this: RSC Adv., 2019, 9, 36351

Received 22nd June 2019

Accepted 24th October 2019

DOI: $10.1039 / c 9 r a 04687 a$

rsc.li/rsc-advances

\title{
Sodium citrate doped polypyrrole/PS glass capillary tube sensor for ultra-small volume $\mathrm{HCl}$ gas detection
}

\author{
Yuxi Chen, ${ }^{\text {ab }}$ Wenqian Zhang, ${ }^{\text {ab }}$ Changkun She, ${ }^{a}$ Guishun Li, ${ }^{\text {ab }}$ Lihua Zhang, ${ }^{\text {ab }}$ \\ Shaohua Liu, (D) a Ya Cheng, ${ }^{c}$ Chengbin Jing (DD *ab and Junhao Chu ${ }^{\mathrm{ab}}$
}

\begin{abstract}
A sodium citrate (SC) doped polypyrrole (PPy)/PS capillary sensor was prepared for ultra-small volume $\mathrm{HCl}$ gas detection. A PS film was formed in advance on the inner wall of a silica glass capillary tube, which enabled us to prepare a high-qualified PPy sensitive film inside the tube. The crystallinity, morphology, microstructure and carrier transport properties of the PPy film were characterized by XRD, SEM, FTIR and Hall effect system, respectively. The results indicated that the as-prepared tube sensor sample was able to detect $0.2 \mathrm{~mL} 30 \mathrm{ppm} \mathrm{HCl}$ gas while the plane-shaped PPy/PS sensor failed to probe. The improvement of sensing properties was attributed to the trend of crystallinity, pore (or gap) morphology and the long-narrow gas cell. The tube-like gas cell and the featured PPy/PS film of the tube sample contribute to sensing the small volume of $\mathrm{HCl}$ gas, which may be applied in breath analysis for potential nonintrusive disease diagnosis.
\end{abstract}

\section{Introduction}

Conductive polymers such as polypyrrole (PPy), polyaniline, and polythiophene have attracted extensive attention, with conductivity attributed to the conjugated $\pi-\pi$ bonds on their backbones. Based on this unique property, they are widely applied in energy, electrical devices, ${ }^{1}$ optical devices, ${ }^{2}$ molecular devices, ${ }^{3}$ electromagnetic interference shielding ${ }^{4}$ and sensors. ${ }^{5}$ As a typical example, PPy is generally excellent as it benefits from a small band gap, adjustable electrical conductivity and environmental stability. ${ }^{6,7}$ At present, most of investigations on PPy are on its application in supercapacitors ${ }^{8}$ and batteries. ${ }^{9}$ In comparison, application in sensors based on PPy is relatively less studied, although PPy is sensitive to specific chemical/ biological species..$^{10}$

Since the PPy sensitive material has electrochemical redox properties and proton acid doping behaviours, the electrical properties of PPy membrane may cause changes upon contacting with several acidity or chemical gases. ${ }^{11-13}$ Therefore, it can be fabricated into gas sensors for the detection of inorganic

${ }^{a}$ Engineering Research Center for Nanophotonics and Advanced Instrument, Ministry of Education, Department of Materials, School of Physics and Electronic Science, East China Normal University, 500 Dongchuan Road, Shanghai 200241, China. E-mail: cbjing@ee.ecnu.edu.cn

${ }^{b}$ Key Laboratory of Polar Materials and Devices, Ministry of Education, Department of Materials, School of Physics and Electronic Science, East China Normal University, 500 Dongchuan Road, Shanghai 200241, China

'The Extreme Optoelectromechanics Laboratory, Department of Materials, School of Physics and Electronic Science, East China Normal University, 500 Dongchuan Road, Shanghai 200241, China and organic gases in environment. ${ }^{14}$ When the PPy membrane adsorbs specific gases, the polymer chain will swell or undergo some kind of chemical reaction, which will affect the electron density of PPy molecular chain and change the conductivity. ${ }^{15,16}$

Based on the above unique mechanism, recently, Jing-Shan Do $e t$ al. proposed a conductimetric acetone gas sensor based on PPy with the high sensitivity and short response time. ${ }^{17}$ In addition, Jeon et al. fabricated the ultra-thin PPy nanosheets for detecting $\mathrm{HCl}$ and $\mathrm{NH}_{3} \cdot{ }^{18}$ In their case, the hydrated $\mathrm{C}_{10} \mathrm{SO}_{3} \mathrm{Na}$ crystals were used as a template to grow PPy nanosheets with layer thickness of $\sim 21 \mathrm{~nm}$ for detecting $\mathrm{HCl}$ and $\mathrm{NH}_{3}$ gases ranging from 20 to $100 \mathrm{ppm}$.

It is well-known that $\mathrm{HCl}$ as a typically toxic gas will cause serious harm and even endanger personal safety once leaked in chemical industry production processes. Furthermore, $\mathrm{HCl}$ is also the main component of human gastric juice, and the changes of chemical properties will be closely related to human health. ${ }^{19,20}$ At present, there has been remarkable growth in the improvement of detecting gaseous $\mathrm{HCl}$ with different sensing materials, including lanthanide complexes, ${ }^{21}$ metal oxides ${ }^{22}$ and conducting polymers. ${ }^{23}$ Currently, Lee and co-authors designed the stimuli-responsive colorimetric and fluorometric sensors based on polydiacetylenes for detecting the $0.8 \mu \mathrm{L}$ of $\mathrm{HCl}$ in a $100 \mathrm{~mL}$ chamber by observing the color change.

Although great progresses have been made in sensing $\mathrm{HCl}$ gas, ${ }^{24}$ there are still several shortcomings. For instance, the volume of most sensing cavity cells is large and it is difficult to detect ultra-small volume gas. For narrowing the cavity cell, hollow optical fiber gas sensors with a long path cell were developed based on the principle that the differences in light 
absorption by different gases. ${ }^{25}$ However, the devices of these optical sensors may be complicated which equipped with expensive laser sources and detector. For the sensing of ultrasmall volume gas, the shapes of previous sensing materials were powders or flat films, probably failing to detect a small amount of gas. Generally, ultra-small volume gas sensing is critical to the application in detecting gases related in human bodies. As far as we know, almost 3500 chemicals are reported in exhaled human breath, ${ }^{26}$ gaseous $\mathrm{HCl}$ may be one of them as it is the main component of human gastric juice. Once the person suffers from stomach disease, the nature of the exhaled $\mathrm{HCl}$ gas by the human body may change. In some emergency situations, only a small volume of breathing gas may be collected for detecting. Therefore, the detection of such small volume gas is an urgent need and still a great challenge.

In this work, a PPy doped sodium citrate (SC) film based on PS/silica capillary tube sensor was proposed and fabricated. It can be expected that the SC serves as a dopant to increase conductivity by releasing protons. For improving the microstructure and conductivity of PPy film, we explored the preparation parameters including the volume ratio of reaction monomer and initiator, reaction time and extraction time. SEM, FTIR, XRD and Hall Effect system were taken to measure the morphology, microstructure and transport properties of samples. To verify the sensing properties of tube sample, we investigated the sensitivity, response time and recover time depending on the tube lengths and concentrations for detecting ultra-small volume $\mathrm{HCl}$ gas $(0.2 \mathrm{~mL})$. In addition, the continuous dynamic response-recovery performance was also measured with the gas volume of $0.2 \mathrm{~mL}$ and concentration of $100 \mathrm{ppm}$. The results indicated that this simple and costeffective tube sensor present the excellent properties in detecting the small volume gaseous $\mathrm{HCl}$.

\section{Experiment}

\subsection{Materials}

Pyrrole (Py, 99\%, MW 67.09, Adamas-beta), ferric chloride hexahydrate $\left(\mathrm{FeCl}_{3} \cdot 6 \mathrm{H}_{2} \mathrm{O},>99 \%\right.$, General-Reagent); sodium citrate tribasic dihydrate (SC, $>99 \%$, General-Reagent), polystyrene (PS, >99\%, Sinopharm Chemical Reagent Co., Ltd), chloroform $\left(\mathrm{CHCl}_{3},>99 \%\right.$, Sinopharm Chemical Reagent Co., Ltd), conductive silver adhesive (HumISEAI, USA), hydrochloric acid ( $\mathrm{HCl},>99 \%$, Jiangsu Qiangsheng), polyimide coated glass capillary tubes $(530 \mu \mathrm{m}, \mathrm{RunTu})$.

\subsection{Fabrications of glass capillary tube and plane-shaped sensor}

In order to grow the PS film in tube, the capillary tube was cleaned by deionized water and ethanol successively before use. Then, 3\% PS/chloroform solution was pumped into glass capillary tube at the flow rate of $0.027 \mathrm{~mL} \mathrm{~min}^{-1}$ controlled by peristaltic pump. PS/chloroform solution remained in the tube for $2 \mathrm{~min}$ then removed out at the same rate of $0.027 \mathrm{~mL} \mathrm{~min}^{-1}$. Subsequently, the tube was dried by pumping air to volatilize the residual chloroform. Finally, white PS film was coated in the inner wall of capillary.

PPy was synthesized in tube by chemical polymerization method at room temperature under atmospheric. $\mathrm{FeCl}_{3} \cdot 6 \mathrm{H}_{2} \mathrm{O}$ $(7.817 \mathrm{~g})$ as the initiator was resolved into $100 \mathrm{~mL}$ deionized water. The dopant SC (0.0369 g) was added into $10 \mathrm{~mL} \mathrm{FeCl}$ solution and stirred until dissolved completely. Then, $100 \mu \mathrm{L}$ Py monomer was reacted with the above initiator solution. After 2 min aging time, the pre-mixed reaction solution was pumped into the above PS coated capillary tube until filled absolutely at the speed of $0.027 \mathrm{~mL} \mathrm{~min}^{-1}$. The static process sustained $1 \mathrm{~h}$ to promote the growth of PPy film. After then, deionized water was pumped to clean the large particles of film surface on the inner wall, followed by dried for $6 \mathrm{~h}$. The schematic diagram of prepared PPy-SC/PS tube sensor is shown in Fig. 1(a).

For comparison, the PPy was grown on both PS and glass plane in the shape of $2 \times 5 \mathrm{~cm}^{2}$, respectively. They were fabricated in the same preparation method, except that the substrates were soaked into the reaction solution instead of using peristaltic pump. The schematic diagram of prepared PPy-SC/PS plane sensor is shown in Fig. 1(b).

\subsection{Characterizations}

In order to investigate the crystal structure of the as-prepared films, some portions were cut from tube and plane samples to be measured using a rotating copper anode X-ray diffractometer (XRD: Rigaku Ultima IV) under $35 \mathrm{kV}$ and $20 \mathrm{~mA}$ irradiation. The scanning electron microscope (SEM: HITACHI S-4800) was used to measure the surface morphology and thickness of the samples in an acceleration voltage of $15 \mathrm{kV}$. The composition and structure of PPy were determined by using a Bruker $80 \mathrm{~V}$ Fourier Transform Infrared Spectrometer (FTIR). The Hall effect test system (HED: Oriental Morning View RCS-80-1) machine was used to test the resistivity, carrier concentration and electronic mobility.

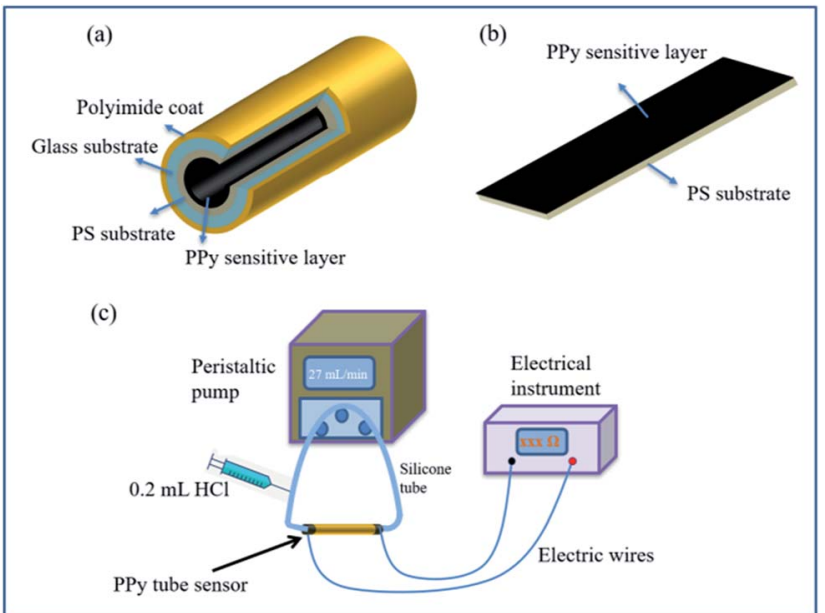

Fig. 1 Schematic diagram of the proposed sensors (a) PPy-SC/PS compound tube and (b) plane sensor. (c) Experimental setup for the $\mathrm{HCl}$ gas detection of PPy-SC/PS tube. 
The experimental setup for the $\mathrm{HCl}$ gas detection of PPy-SC/ PS tube was shown in Fig. 1(c). $\mathrm{HCl}$ gas was collected from the vapor of hydrochloric acid solution. Then we used pure $\mathrm{N}_{2}$ gas to dilute above gas to obtain different concentrations of $\mathrm{HCl}$ gas. $\mathrm{HCl}$ gas was collected from the vapor of hydrochloric acid solution. Then we used pure $\mathrm{N}_{2}$ gas to dilute above gas to obtain different concentrations of $\mathrm{HCl}$ gas. The concentration based on the formula $V_{\mathrm{x}}=V \times C \times 10^{3} \times \frac{273+T_{\mathrm{r}}}{273+T_{\mathrm{b}}}$, where $V_{\mathrm{x}}$ is actual volume of gas injection, $V$ is gas chamber volume, $C$ (ppm) is initial gas concentration, $T_{\mathrm{r}}$ is room temperature, $T_{\mathrm{b}}$ is the temperature in gas chamber and the final concentration is also measured by commercial detector (ADKS-1, Aidekesi Company). The $0.2 \mathrm{~mL}$ mixed $\mathrm{N}_{2} / \mathrm{HCl}$ gases were injected into the tube controlled by syringe. Both ends of the PPy-SC/PS tube were coated with silver glue and wound with copper wires as electrodes. The changed electrical signals depending on $\mathrm{HCl}$ gas were recorded by electrical instrument. All experiments were operated at room temperature $\left(25.0{ }^{\circ} \mathrm{C}\right)$ and the relative humidity (60\% RH).

\section{Results and discussion}

In our initial experiments, silica glass was chosen as substrate to grow PPy sensitive film. Unfortunately, we found that only few PPy particles were grown on the glass plane and easy to peel off upon cleaning by deionized water. Generally speaking, PS polymer can be as an excellent alternative substrate owing to its negatively charged groups. In view of this, a compound structure of PS/glass capillary tube is proposed to fabricate PPy tube sensor for small volume gas.

We conducted many experiments to explore the optimum film formation parameters. It can be found that the aging time of precursor solution has a great influence on the film growth morphology because the formation rate of PPy particles is relatively fast, resulting in a cotton-like coarse film. Take that into account, an incompletely reacted precursor solution was pumped into the tube for improving the quality of PPy film onto the inner wall of the tube. Also, the influence of growing time was explored systemically which may affect the thickness and initial resistance of PPy film. The results indicated that the PPy has the minimum resistance with the aging time of $2 \mathrm{~min}$ and the growing time of $1 \mathrm{~h}$.

Fig. 2 shows the X-ray diffraction patterns of as-prepared $\mathrm{PPy}-\mathrm{SC} / \mathrm{PS}$ in the tube and on the plane, respectively. There are obvious wide peaks at about $2 \theta=24.3^{\circ}$ in both tube and plane samples indicating the amorphous structure of PPy, which almost match to the distance of the interplanar aromatic rings. ${ }^{27}$ However, the tube sample holds stronger peak intensity and narrower full width at half maximum revealing that the PPy chains of tube sample may be located in a more ordered state with better trend of crystallinity. ${ }^{28}$

Fig. 3(a)-(c) shows SEM images of PPy-SC on different substrates (a) glass plane, (b) PS plane, (c) PS tube, respectively. It can be seen from Fig. 3(a) that only a few PPy particles grow on the glass substrate, indicating that PPy is difficult to form a film on the smooth glass due to weak interaction force at the

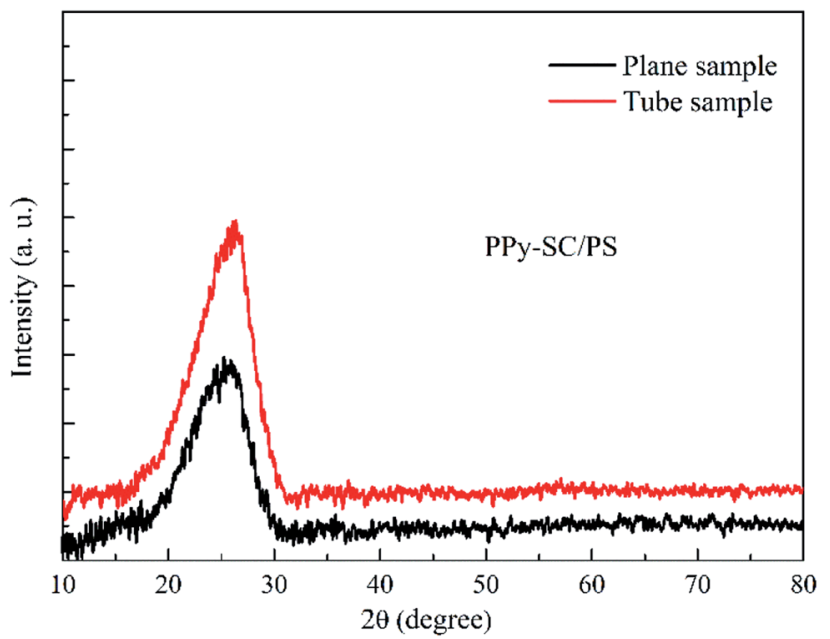

Fig. 2 The XRD patterns of PPy-SC/PS plane and tube samples, respectively.

interface. The results are consistent with the above experimental phenomena. In comparison, continuous and stacked PPy can be found when growing on the PS substrate as shown in Fig. 3(b) and (c). There are a larger number of PPy particles with a diameter of about $200 \mathrm{~nm}$ and the pore size of about $2 \mu \mathrm{m}$. These pores may result from not fully dense agglomeration in PPy growing process. It should be noted that the pore structure possesses the large the surface-area-to-volume ratio which may contribute to the gas sensing. Moreover, the higher degree of dense agglomeration can be observed in PPy grown on PS tube substrate (Fig. 3(c)) compared with the PS plane sample (Fig. 3(b)).

It is still difficult for us to know if there are certain mechanisms of PPy growth on the inner wall of tube. We conclude that PPy particles will adhere to the inner wall of the glass tube to form a thin film at the beginning of growth.$^{29}$ It is worth noting that the glass tube has a small radius of curvature, and the growth direction of PPy anywhere is directed to the tube centre.

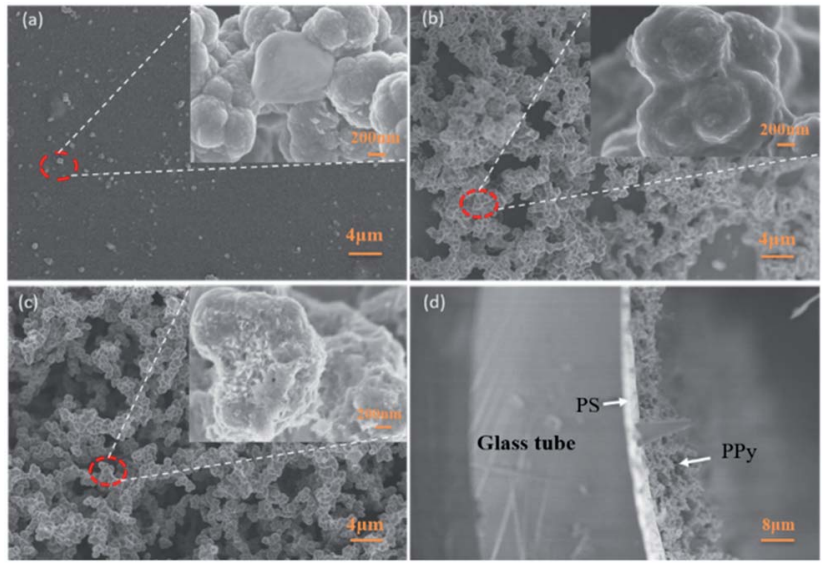

Fig. 3 SEM images of PPy-SC on different substrates (a) glass plane, (b) PS plane, (c) PS tube, (d) the cross-section of PS tube sample. Note: the inset is an amplification of circled place. 


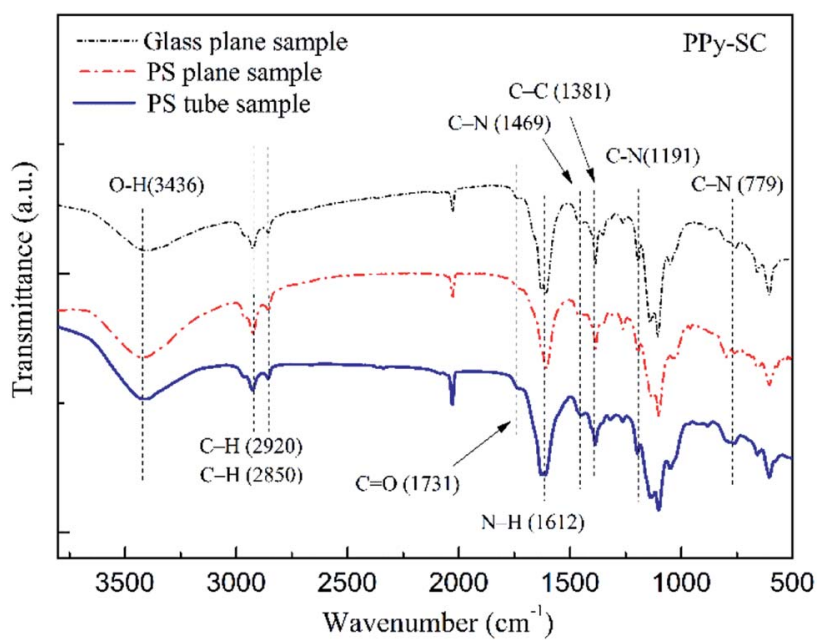

Fig. 4 The FTIR spectrum of PPy-SC on different substrates (tube, glass tube and PS plane, respectively).

Herein, this growth orientation may promote the agglomeration of PPy during the growth process. Further studies are underway. The part cross-sectional morphology of PPy-SC/PS compound tube sample is displayed in Fig. 2(d). The clear structure of 4 layers can be seen, in which the thicknesses of the PS and PPy layer are about $1 \mu \mathrm{m}$ and $700 \mathrm{~nm}$, respectively.

The FTIR spectrum of PPy-SC on different substrates are shown in Fig. 4. It can be found that five-membered ringstretching and conjugated $\mathrm{C}-\mathrm{N}$ stretching vibration peaks in PPy at $1612 \mathrm{~cm}^{-1}$ and $1469 \mathrm{~cm}^{-1}$, respectively. ${ }^{30}$ The peaks at $779 \mathrm{~cm}^{-1}$ and $1191 \mathrm{~cm}^{-1}$ are associated with the wagging and in-plane vibration. In addition, the bands at both $2920 \mathrm{~cm}^{-1}$ and $2850 \mathrm{~cm}^{-1}$ corresponded to five membered ring $\mathrm{C}-\mathrm{H}$ stretching. These main peaks are overlap perfectly in three curves, indicating that the PPy has been synthesized successfully.

In order to investigate the electrical properties of PPy-SC, we measure the specific resistance, electronic mobility ratio and carrier concentration by Hall effect system as depicted in Table 1. It is found that the PS plane sample has lower specific resistance, electronic mobility ratio and higher carrier concentration. The carrier concentration value of PS sample is 3 times that of glass sample. Herein, PPy-SC/PS sample possesses more excellent electronic conductivity which may be beneficial for gas sensing.

In order to investigate the electrical properties of PPy-SC, we measure the specific resistance, electronic mobility ratio and carrier concentration by Hall effect system as depicted in Table 1. A Hall instrument was used to measure the carrier concentration $n$ and mobility $\mu$ of the sample. The $n$ and $\mu$ values can be estimated based on formulas $1 /(n q)=V_{\mathrm{H}} t /(I B)$ and $\mu=V_{\mathrm{H}} t /$ $(I B)$, respectively, where $q$ is electron charge, $V_{\mathrm{H}}$ is the Hall voltage, $t$ is the sample thickness, $I$ is the current, $B$ is the magnetic field, $\sigma$ is electrical conductivity. It is found that the PS plane sample has lower specific resistance, electronic mobility ratio and higher carrier concentration. The carrier concentration value of PS sample is 3 times that of glass sample. Herein, PPy-SC/PS sample possesses more excellent electronic conductivity which may be beneficial for gas sensing.

The sensitivity $S$ is defined as $S=\left(R-R_{0}\right) / R_{0} \times 100 \%$, where $R$ is the measured real-time resistance and $R_{0}$ is the initial resistance. ${ }^{31}$ The response time is defined as the time corresponding that resistance value reaches to $90 \%$ of its change in presence of the test gas. The recovery time is defined as the time back to $90 \%$ of resistance change. In order to prove the effectiveness of the proposed capillary sensor for the detection of ultra-small volume gases, sensing performances of three PPy-SC samples (PS tube, glass plane and PS plane) were compared and discussed in this work.

The influences of different substrates on the sensing performances such as response time, recovery time and sensitivity are investigated with $0.2 \mathrm{~mL}$ volume $\mathrm{HCl}$ gas $(200 \mathrm{ppm})$ at the flow rate of $27 \mathrm{~mL} \mathrm{~min}{ }^{-1}$, as shown in Fig. 5. We calculated the sensitivities, response times and recovery times of PPy-SC samples with the substrate of PS tube $(12.9 \%, 18 \mathrm{~s}, 77 \mathrm{~s})$, PS plane $(3.2 \%, 10 \mathrm{~s}, 10 \mathrm{~s})$ and glass plane $(0.2 \%, 6 \mathrm{~s}, 8 \mathrm{~s})$, respectively. The tube sensor with PS film holds higher

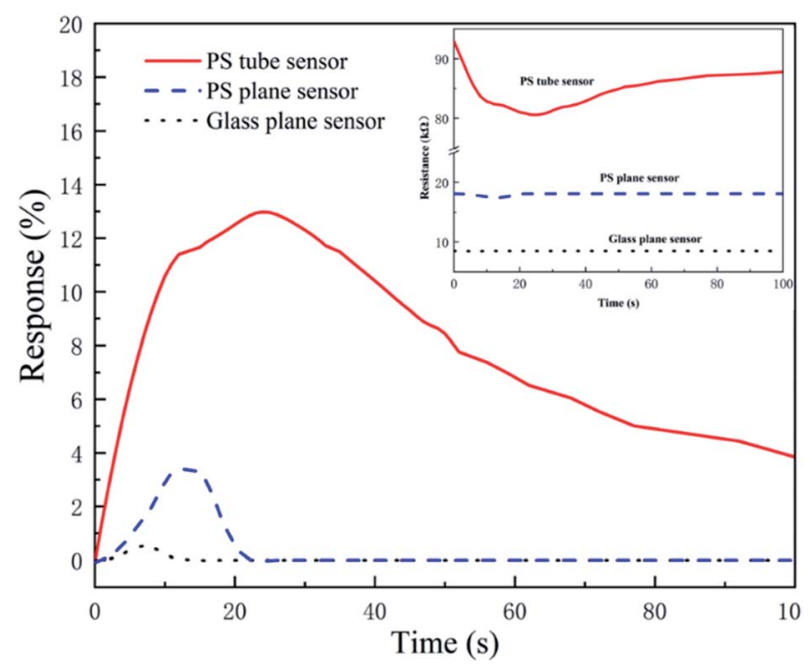

Fig. 5 The transient responses of PPy-SC sensors with different substrates (PS tube, glass plane and PS plane) exposed to $0.2 \mathrm{~mL}$, $100 \mathrm{ppm} \mathrm{HCl}$ gas. The inset: the changes of corresponding real-time resistance.

Table 1 The electrical parameters of PPy-SC/glass and PPy-SC/PS samples

\begin{tabular}{lllll}
\hline Sample & Type & Specific resistance $(\mathrm{ohm} \mathrm{m})$ & Mobility ratio $\left(\mathrm{m}^{2}(\mathrm{~V} \mathrm{~s})^{-1}\right)$ & ${\text { Carrier concentration }\left(\mathrm{m}^{-3}\right)}^{-1}$ \\
\hline PPy-SC/glass & $\mathrm{p}$ & $1.99455 \times 10^{-3}$ & $1.18033 \times 10^{-4}$ & $2.65120 \times 10^{25}$ \\
PPy-SC/PS & $\mathrm{p}$ & $7.87441 \times 10^{-4}$ & $1.04687 \times 10^{-4}$ & $7.57147 \times 10^{25}$
\end{tabular}


sensitivity in spite of longer response time and recovery time. It is reasonable that there are more sensitive particles, namely larger surface area-to-volume ratio and pore structure in PPy-SC/ PS tube sample comparing with plane samples. Furthermore, tube sensor has a long-narrow gas cell, which brings more $\mathrm{HCl}$ gas to sufficiently react with PPy in tube. It is revealed that the tube sensor has a significant advantage in detecting small volumes of $\mathrm{HCl}$ gas, such as $0.2 \mathrm{~mL}$ ultra-small volume gas.

Fig. 6 presents the transient responses of PPy-SC/PS tube sensor with the length of 3 and $5 \mathrm{~cm}(0.2 \mathrm{~mL}, 300 \mathrm{ppm}$ and 27 $\left.\mathrm{mL} \min ^{-1}\right)$. The sensitivity of $5 \mathrm{~cm}$ tube sensor is $26.3 \%$ higher than that of $3 \mathrm{~cm}$ tube sensor $(21.8 \%)$. The response time and recovery time of tube sensor are also calculated with the length of $5 \mathrm{~cm}$ tube $(81 \mathrm{~s}, 670 \mathrm{~s})$ and $3 \mathrm{~cm}$ tube $(20 \mathrm{~s}, 96 \mathrm{~s})$. On one hand, after removing $\mathrm{HCl}$, a small amount of $\mathrm{HCl}$ molecules are still adsorbed by the surface molecules of PPy, so the full recovery may occur after sufficient time. On the other hand, it is reported that many types of polymer tubes possess strong gas adsorption capacity. ${ }^{32}$ We noticed that our tube sensor connects with silicone tube to make up a closed loop system. The desorbed $\mathrm{HCl}$ molecules from PPy may be captured by the inner wall of silicone tube. It may also impose influence on the recovery of the sensor. Future research is needed to improve this. Although, the shorter PPy-SC/PS tube sample holds shorter response time and recovery time, the longer sample shows higher sensitivity. The long-narrow gas cell can bring dynamically molecular collisions in $\mathrm{HCl}$ molecule and PPy polymer as gas flows in it, which contributes to the enhancement of effective contact area between them. It is understandable that a longer tube sample has higher sensitivity and longer recovery time. For the reason that, short length of PPy-SC/PS tube sensor is a good choice for fast response and recovery time while long length sample is effective for high sensitivity. Thus, $5 \mathrm{~cm}$ tube sensor is better choice for ultra-small volume sensing.

The transient responses of PPy-SC/PS capillary tube sensor to various gas concentrations ( $5 \mathrm{~cm}$ length, $0.2 \mathrm{~mL} \mathrm{HCl}$ ), as shown

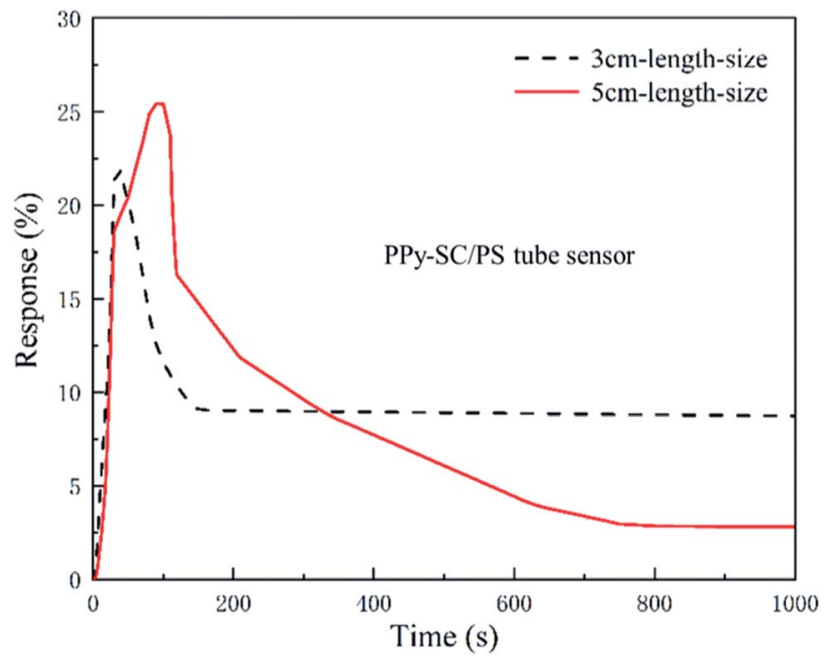

Fig. 6 The transient responses of PPy-SC/PS tube sensor with the length of 3 and $5 \mathrm{~cm}$.

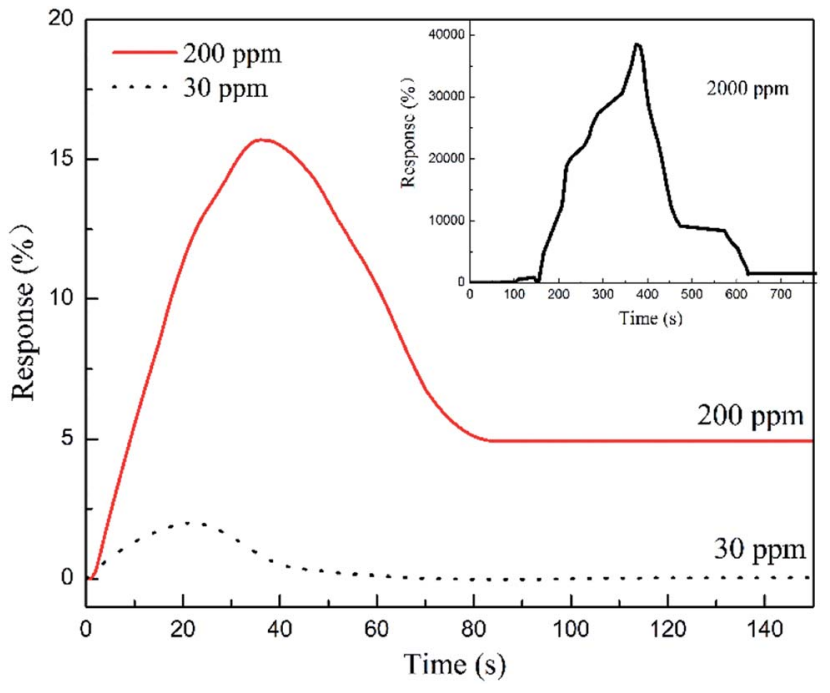

Fig. 7 The transient responses of PPy-SC/PS capillary tube sensor under different concentration (30 and $200 \mathrm{ppm}$ ) of $0.2 \mathrm{~mL} \mathrm{HCl}$ gas. The inset is the transient response with a concentration at $2000 \mathrm{ppm}$.

in Fig. 7. As the $\mathrm{HCl}$ gas concentration increasing from 30 to $2000 \mathrm{ppm}$, all of sensitivity response time and recovery time appear the increasing trends from $30 \mathrm{ppm}(1.9 \%, 18 \mathrm{~s}, 23 \mathrm{~s})$ to $2000 \mathrm{ppm}$ (38 503\%, $356 \mathrm{~s}, 199 \mathrm{~s})$. It is worth noting that for the plane sensors, there is no obvious resistance change when detecting $30 \mathrm{ppm} \mathrm{HCl}$ gas. This further illustrates that the tube sensor is reliable for detecting low concentration with small volume $\mathrm{HCl}$ gas. Generally, a large gas chamber with a volume of about $20 \mathrm{~L}$ has been used for measurement of the sensing performances of a traditional plane sensor sample. It is inconvenient in some cases where a small volume of gas sample is available, such as medical respiratory gas detection. In this sense, the capillary tube-like sensor is advantageous since it needs only a small volume of gas sample. Besides the $100 \mathrm{ppm}$ $\mathrm{HCl}$ gas, we also measured the sensing property of the tube sensor for $30 \mathrm{ppm} \mathrm{HCl}$ gas sample. The response reaches 1.9\% and the response time is $18 \mathrm{~s}$ (see Fig. 7). We noticed that there are few reports on ultra-low concentration $\mathrm{HCl}$ gas detection, although PPy has been used to detect a $20 \mathrm{ppm} \mathrm{HCl}$ gas sample (response is $30 \%) .{ }^{15}$ Future work is needed to further improve the sensing performance of the tube-like sensor.

To investigate the response and the recovery properties, Fig. 8(a) presents the four reproducibility tests of the $5 \mathrm{~cm}$ length PPy-SC/PS tube sensor exposed to $0.2 \mathrm{~mL}, 100 \mathrm{ppm}$ $\mathrm{HCl}$ gas. As shown in the inset of Fig. 8(a), initially, the resistance value decreases rapidly upon pumping $\mathrm{HCl}$ gas, subsequently, the resistance recovers slowly after the removal of $\mathrm{HCl}$ gas and pumping air gas. The results show that PPy-SC/PS tube sensor possesses outstanding reproducibility performance although it may take very long time to recover absolutely. The long-term stability was investigated by monitoring the sensing response per $12 \mathrm{~h}$ exposed to $100 \mathrm{ppm}$ of $\mathrm{HCl}$ gas (see Fig. 8(b)). It can be seen that the sensing performance of this sensor remains almost unchanged. 
(a)

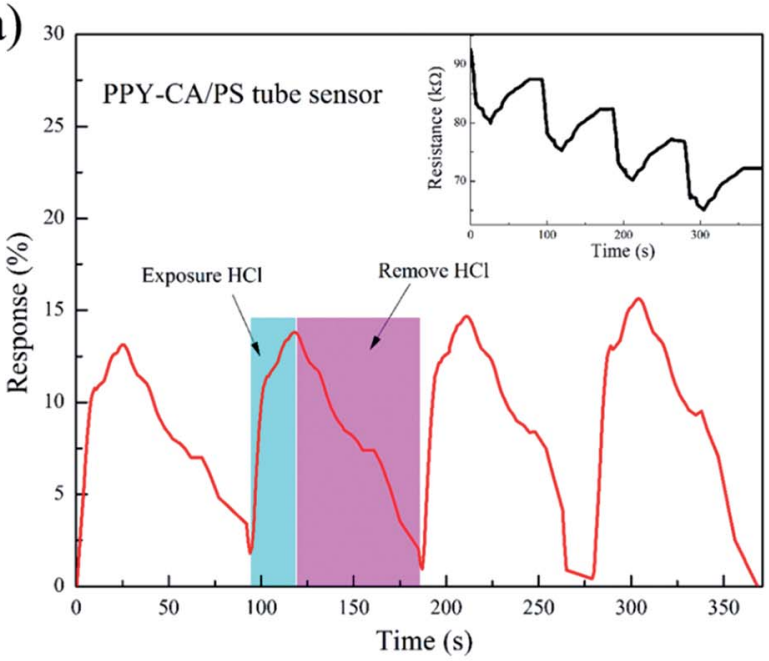

(b)

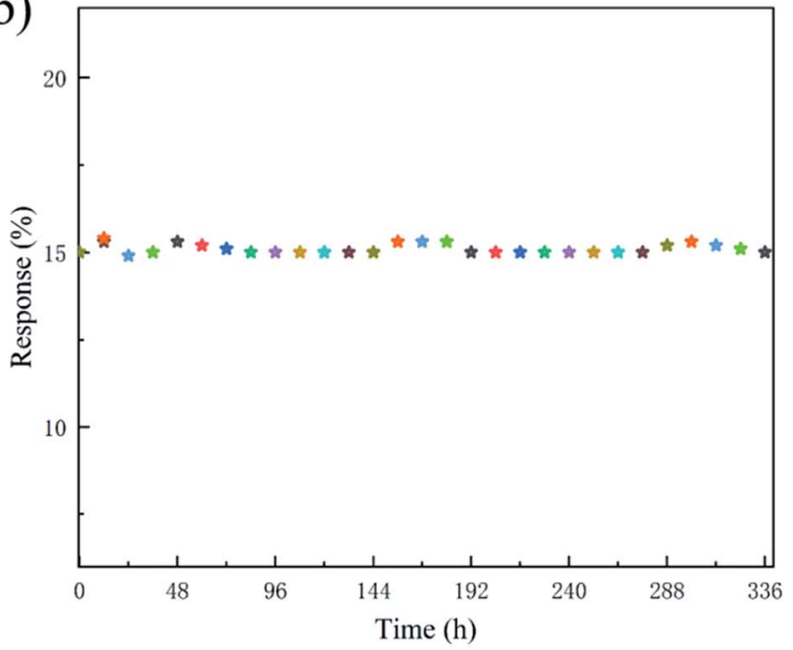

Fig. 8 (a) The reproducibility of the PPy-SC/PS tube sensor depending on $\mathrm{HCl}$ gas $(0.2 \mathrm{~mL}, 100 \mathrm{ppm})$. The inset: the changes of real-time resistance. (b) The long-term stability of the sensor per 12 hours exposed to $100 \mathrm{ppm} \mathrm{HCl}$ and $\mathrm{N}_{2}$ gas.

The sensing mechanism can be explained as follows. ${ }^{15,33}$ The sensing process is connected with the oxidation level of the PPy material. Generally, PPy is a p-type semiconductor in conducting state. Upon the $\mathrm{HCl}$ molecules introduced into PPy, the doping of PPy is achieved by means of the protonation of the amine group by $\mathrm{HCl}$. On the basis of electric neutral balance principle, the PPy backbone would become positively charged by this protonation. In this process, the polarons in the PPy chain promote two localized electronic levels in the band gap including the bonding and anti-bonding cation levels. Moreover, at higher oxidation levels, the polarons associate with each other thereby producing the doubly charged spinless bipolarons. In this case, the oxidation level of PPy is easily affected by the external environment, such as chemical signals and electromagnetic waves, which may cause changes in the conductivity of PPy. The conductivity of PPy changes by the formation of radical cations that travel along the polymer backbone. In other words, the negatively charged $\mathrm{Cl}^{-}$ions are incorporated

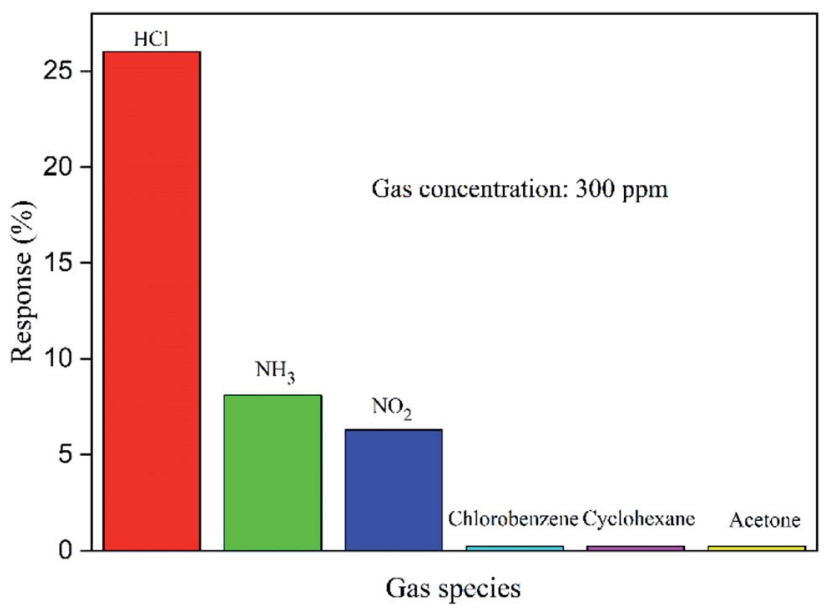

Fig. 9 Selectivity histogram for sensing response of PPy tube sensor with different gases. (Note: concentration of each gas is 300 ppm.)

into the PPy layer, resulting to the increase of electrical conductivity of PPy. So, a drop in resistance can be observed.

The selectivity is an important factor of gas sensors for detecting a specific gas. In order to measure the gas selectivity, we tested the response to several other gases with same concentration (300 ppm), such as $\mathrm{NH}_{3}$, chlorobenzene, $\mathrm{NO}_{2}$, cyclohexane and acetone (see Fig. 9). The results indicate the PPy sensor is rarely sensitive to these gases, and the best response reaches $8.1 \%\left(\mathrm{NH}_{3}\right)$ which is lower than $\mathrm{HCl}(26.3 \%)$. Therefore, this sensor is much more sensitive to $\mathrm{HCl}$ gas.

In fact, the sensing response of the PPy sensor is not very high, especially when compared it to other fluorescent $\mathrm{HCl}$ sensors. $^{34,35}$ However, the designed capillary tube-like PPy sensor is based on the detection of electricity signals instead of fluorescent intensity. It has the advantages of convenient operation, low-cost, device miniaturization, and can be applied to small volume gas detection. This will be able to pave a way for practical applications.

\section{Conclusions}

The PPy-SC/PS tube sensor for ultra-small volume $\mathrm{HCl}$ gas detection has been proposed in this work. The high-qualified PPy sensing film was grown on the surface of PS film which PS deposited previously on the inner wall of a silica glass capillary tube. For enhancing the microstructure and conductivity of PPy film, preparation parameters were optimized with the volume ratio of reaction monomer and initiator of $100: 1$, reaction time of $1 \mathrm{~h}$ and extraction time of $2 \mathrm{~min}$. From the comparative gas sensing property analysis, the results revealed excellent sensor performances for $530 \mu \mathrm{m}$ bore diameter, $5 \mathrm{~cm}$ length PPy-SC/PS tube sensor. This tube sample was able to detect the small volume and low concentration $\mathrm{HCl}$ gas $(0.2 \mathrm{~mL}$, $30 \mathrm{ppm}$ ) with response time of $18 \mathrm{~s}$, sensitivity of $1.9 \%$ while the plane-shaped sensor failed to detect. The improvement of sensing performances was ascribed to the trend of crystallinity, pore (or gap) morphology and long-narrow gas cell. This tube sensor would provide promising application prospects in 
detecting the ultra-small volume $\mathrm{HCl}$ gas, such as medical gas sensing.

\section{Conflicts of interest}

There are no conflicts to declare.

\section{Acknowledgements}

The authors acknowledge the financial support provided within National Natural Science Foundation of China (NSFC) (61775060, 61275100, 61761136006).

\section{References}

1 J. Janata and M. Josowicz, Nat. Mater., 2003, 2, 19.

2 P. Wang, L. Zhang, Y. Xia, L. Tong, X. Xu and Y. Ying, Nano Lett., 2012, 12, 3145.

3 S. Agarwal, A. Greiner and J. H. Wendorff, Prog. Polym. Sci., 2013, 38, 963.

4 G. Kulkarni, P. Kandesar and N. Velhal, Chem. Eng. J., 2019, 355, 196-207.

5 Y. Hu, H. Yu and Z. Yan, RSC Adv., 2018, 16, 8747-8754.

6 D. T. McQude, A. E. Pullen and T. M. Swager, Chem. Rev., 2000, 100, 2537-2574.

7 D. B. Cairns, M. A. Khan, C. Perruchot, A. Riede and S. P. Armes, Chem. Mater., 2003, 15, 233-239.

8 H. Liu, Q. Zhao and K. Wang, RSC Adv., 2019, 12, 6890-6897.

9 R. J. Yang, T. T. Yu and X. Y. Zhao, J. Alloys Compd., 2019, 788, 407-412.

10 R. Zimmerling, U. Dämmgen, A. Küsters, L. Grünhage and H. J. Jäger, Environ. Pollut., 1996, 2, 139-147.

11 Q. Ameer and S. B. Adeloju, Sens. Actuators, B, 2005, 106, 541-552.

12 B. J. Tongol, C. A. Binag and F. B. Sevilla, Sens. Actuators, B, 2003, 93, 187-196.

13 J. H. Cho, J. B. Yu, J. S. Kim, S. O. Sohn, D. D. Lee and J. S. Huh, Sens. Actuators, B, 2005, 108, 389-392.

14 S. T. Navale, A. T. Mane, M. A. Chougule, R. D. Sakhare, S. R. Nalage and V. B. Patil, Synth. Met., 2014, 189, 94-99.
15 J. Jang and J. Bae, Sens. Actuators, B, 2007, 122, 7-13.

16 M. Cano, P. Castillero, J. Roales, J. M. Pedrosa, S. Brittle, T. Richardson, A. R. González-Elipe and A. Barranco, Sens. Actuators, B, 2010, 150, 764-769.

17 J. S. Do and S. H. Wang, Sens. Actuators, B, 2013, 185, 39-46.

18 S. S. Jeon, H. H. An, C. S. Yoon and S. S. Im, Polymer, 2011, 3, 652-657.

19 G. M. Spinks, B. Xi, V. T. Truong and G. G. Wallace, Synth. Met., 2005, 151, 85-91.

20 C. D. Evans, D. T. Monteith, D. Fowler, J. N. Cape and S. Brayshaw, Environ. Sci. Technol., 2011, 45, 1887-1894.

21 Q. F. Li, L. Jin, L. Li, W. Ma, Z. Wang and J. Hao, J. Mater. Chem. C, 2017, 19, 4670-4676.

22 M. L. Yin, L. M. Yu and S. Z. Liu, J. Alloys Compd., 2017, 696, 490-497.

23 X. Wang, S. Meng, W. Ma, J. Pionteck, M. Gnanaseelan, Z. Zhou and M. Zhu, React. Funct. Polym., 2017, 112, 74-80.

24 S. K. Tripathy, J. Y. Woo and C.-S. Han, Anal. Chem., 2011, 83, 9206-9212.

25 T. Katagiri, T. Suzuki and Y. Matsuura, Opt. Eng., 2018, 5, 054-104.

26 M. Phillips, J. Herrera and S. Krishnan, J. Chromatogr. B: Biomed. Sci. Appl., 1999, 729, 75-88.

27 J. Sun, X. Shu, Y. Tian, Z. Tong, S. Bai, R. Luo, D. Li and C. C. Liu, Sens. Actuators, B, 2017, 241, 658-664.

28 X. Yang, L. Li and Y. Zhao, Synth. Met., 2010, 160, 1822-1825.

29 Y. Gao, S. Yao, J. Gong and L. Qu, Macromol. Rapid Commun., 2007, 3, 286-291.

30 K. Firoz Babu, P. Dhandapani, S. Maruthamuthu and M. Anbu Kulandainathan, Polymer, 2012, 90, 1557-1563.

31 E. H. Cordes and W. P. Jencks, J. Am. Chem. Soc., 1962, 84, 832-837.

32 Z. Zhu, Appl. Eng. Agric., 2012, 28, 265-269.

33 S. Virji, J. Huang and R. B. Kaner, Nano Lett., 2004, 4, 491496.

34 M. Hu, W. Kang, Y. Zhao, J. Shi and B. Cheng, RSC Adv., 2017, 7, 26849-26856.

35 M. Hu, W. Kang, Z. Zhong, B. Cheng and W. Xing, Ind. Eng. Chem. Res., 2018, 57, 11668-11674. 